\title{
Stress and neuroimmune regulation of gut mucosal function
}

\author{
J Santos, M H Perdue
}

\section{Department of Pathology and Molecular Medicine, McMaster University, HSC-3NC, Room 3N5C, 1200 Main St West, Hamilton, Ontario, Canada J Santos M H Perdue}

Correspondence to: Dr J Santos. jsantos@hg.vhebron.es
The intestinal mucosa is composed of three layers: the innermost muscularis mucosae, lamina propria, and the epithelial layer which is exposed to the lumen. Besides being the structural support for the epithelium, the lamina propria contains elements important for immunological and absorptive functions. Thus in the lamina propria we find blood and lymph vessels, nerve varicosities, and a plethora of immune cells (plasma cells, lymphocytes, macrophages, eosinophils, mast cells, dendritic cells, etc). ${ }^{1}$ The lamina propria is densely innervated via both intrinsic (mainly from the submucosal plexus) and extrinsic nerve fibres. From the lamina propria, chemical messengers from nerves and immune cells reach and act on epithelial cell receptors.

The intestinal epithelium is a single layer of columnar cells. The most common cells are the enterocytes (absorptive cells). However, a rich variety of cells are also present, including goblet cells (secrete mucus), endocrine cells (secrete hormones and neuropeptides), Paneth cells, caveolated (tuft) and cup cells, $M$ cells,

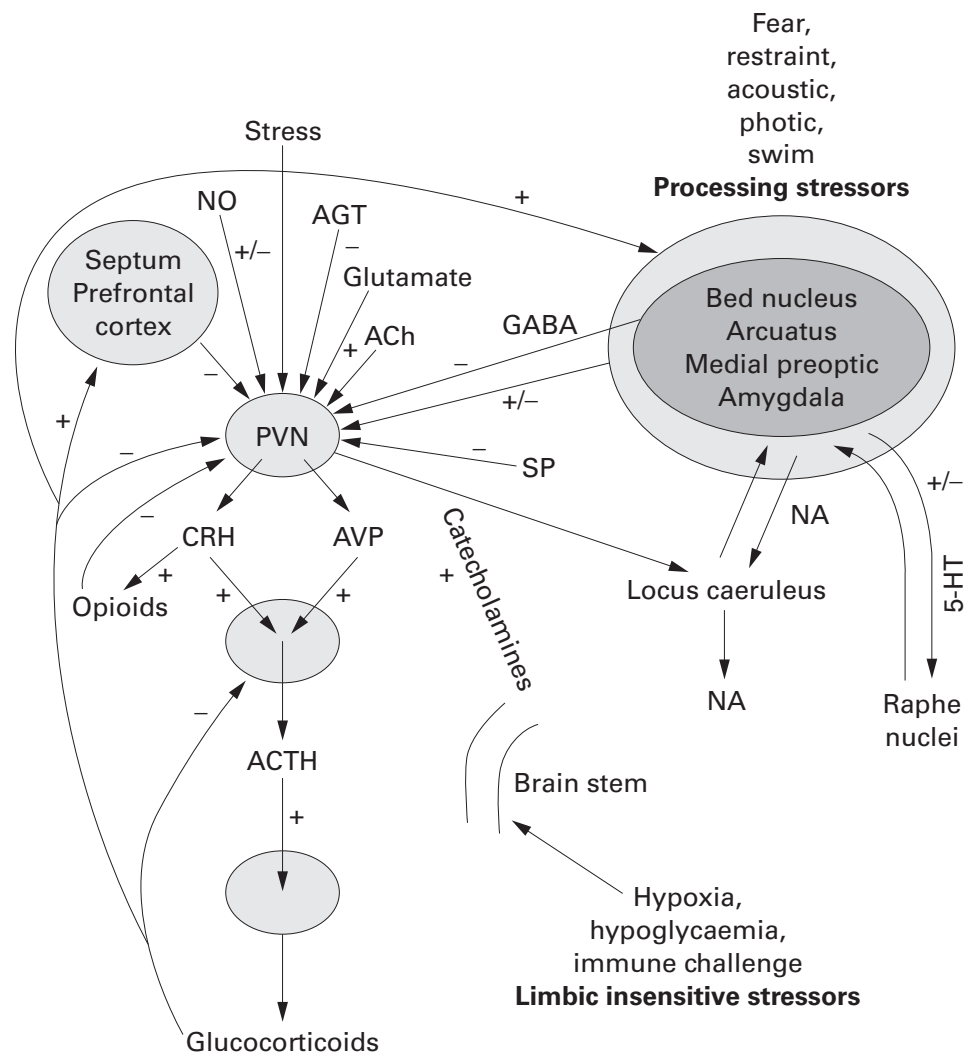

Figure 1 Regulation of intestinal secretion and permeability. SP, substance P; CRH, corticotrophin releasing hormone; $A C h$, acetylcholine; $N A$, noradrenaline; $N O$, nitric oxide; AVP, arginine vasopressin; 5-HT, 5-hydroxytryptamine; GABA, gamma amino butyric acid; $A T C H$, adrenocorticotrophic hormone; AGT, angiotensin; $P V N$, paraventricular nucleus. stem cells (crypt), and intraepithelial lymphocytes. Epithelial cells are bound together by junctional complexes in the apical membrane ${ }^{1}$ : the tight junction (zona occludens), the intermediate junction (zonula adherens), and the desmosomes (macula adherens). These structures define the paracellular space.

The epithelial lining serves several functions in the intestinal tract. ${ }^{2}$ The junctional complex acts as a physical barrier limiting the uptake of microbial and food derived antigens. However, in certain conditions macromolecules and even cells (mainly neutrophils) may be able to cross it to reach the lamina propria or the luminal surface, respectively. The presence of various enzymes on the apical surface and within epithelial cells contributes to the digestion and absorption of nutrients as well as to the degradation of immunogenic particles. Absorption and secretion of water and electrolytes is regulated by the epithelium. The epithelium maintains its own integrity through continuous cellular turnover derived from stem cells in the crypts of Lieberkühn. Finally, the epithelium plays an active role in immunological and inflammatory events, acting as an antigen presenting system (expressing major histocompatibility antigens), expressing adhesion molecules, and releasing cytokines, chemokines, and other regulatory molecules that participate in the trafficking and homing of immune cells in the gut.

Intestinal secretion and permeability are regulated by neural and immune factors (fig 1). These aspects have been extensively documented using several models of intestinal anaphylaxis. ${ }^{2}$ Neural regulation of mucosal functions has been the subject of previous talks in this symposium. This paper will focus on the immune regulation.

Regulation of ion transport by immune factors can be exemplified by the role of mast cells in rodent models of intestinal hypersensitivity. Typically, intestinal allergic reactions to food antigens are characterised by increased water and ion secretion and enhanced permeability. Mast cells contain a vast array of molecules, such as histamine, prostaglandins, nitric oxide, or cytokines that can alter epithelial function. Participation of mast cells in these reactions was suggested by the lack of secretory response in sensitised jejunal tissues mounted in Ussing chambers after pretreatment with the mast cell stabiliser doxantrazole. ${ }^{3}$ Studies in sensitised mast cell deficient mice (W/Wv)

Abbreviations used in this paper: SP, substance P; RS, restraint stress; WA, water avoidance; HRP, horseradish peroxidase; CRH, corticotrophin releasing hormone. 


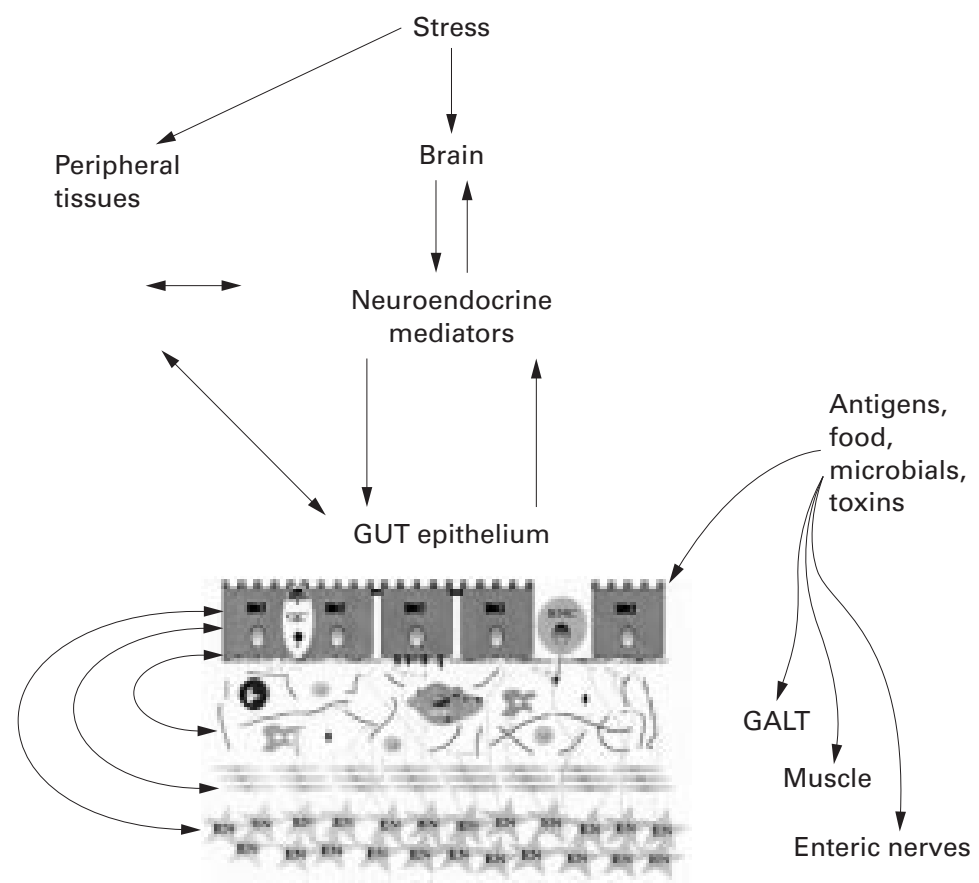

Figure 2 Adaptive responses to stress. GALT, gut associated lymphoid tissue.

provided direct evidence for mast cell involvement in antigen induced epithelial secretion. In these rodents, a $70 \%$ reduction in the secretory response to serosal antigen was shown in comparison with sensitised normal congenic mice. ${ }^{4}$ Moreover, this response was entirely restored after reconstitution of mucosal mast cells by injection of bone marrow derived mast cell precursors. Final support for mast cell involvement in secretory responses was obtained in human colonic epithelial cells (HCA-7). Secretory responses to the antigen ovalbumin were observed only when epithelial cells were sandwiched in Ussing chambers with mast cells sensitised by anti-ovalbumin IgE but not when sandwiched with non-primed mast cells. ${ }^{5}$

Mast cells are also involved in the modulation of epithelial permeability to small and large molecules. Using horseradish peroxidase (HRP) (44 kDa) as a model protein, it has been shown that enhancement of paracellular transepithelial transport of this protein (assessed by electron microscopy) observed in sensitised $+/+$ rats after luminal challenge was not present in sensitised mast cell deficient rats (Ws/Ws) ${ }^{6}$ This indicated the requirement for mast cells for recruitment of the paracellular pathway for transport of macromolecules in the intestine.

Neuroanatomical studies have provided strong evidence for the direct innervation of gastrointestinal mucosal mast cells. Close contacts between mast cells and peptidergic nerves (mainly substance P (SP) containing varicosities) have been reported in the small intestine. ${ }^{7}$ Furthermore, a large list of neuropeptides has been shown to induce rat peritoneal mast cells to secrete histamine and other mediators. In addition, receptors for many of these neuropeptides on mast cells have been demonstrated by binding and electrophysiological studies. However, only some of these neuropeptides, including $\mathrm{SP}$ and calcitonin gene related peptide, have been shown to activate mucosal mast cells.

Mast cell responses to neural stimulation are heterogeneous. While sympathetic activation has been shown to inhibit mast cells, vagal stimulation enhances activation. Moreover, experiments with classical Pavlovian conditioning have shown that activation of mast cells, indicated by serum levels of a specific mast cell protease, occurred after exposure to the conditional stimulus (audiovisual cue). ${ }^{8}$ This activation took place only in sensitised animals in which the conditional stimulus and the unconditional stimulus (antigen challenge) had been paired, but not in animals where both stimuli were unassociated, indicating that mast cell activity is subject to central nervous system modulation.

The functional relevance of mast cell-nerve interactions has been outlined in several models of intestinal hypersensitivity. ${ }^{9}$ Different pharmacological agents (tetrotodoxin, atropine) have shown that neural blockade inhibits secretory responses to antigen challenge. Moreover, blockade of neural activity was only effective in normal mice but not in mast cell deficient littermates. Finally, activation of mast cells induced by Pavlovian conditioning was associated with increased intestinal ion secretion and supramaximal responses to antigen challenge. These findings, taken together, highlight the importance of nerve-mast cell cross talk in the regulation of epithelial responses.

It is clear that the secretory and transport functions of the intestinal epithelium are regulated by neural and immune factors. Adaptive responses to stress comprise a complex network of neural, endocrine, immune, and molecular reactions that purposely aim to maintain physiological and behavioural homeostasis (fig 2). Stress has been associated with reactivation of inflammatory and functional disorders of the small and large intestine..$^{10}$ In humans, physical and psychological stressors have been shown to induce motor and water transport abnormalities in the gastrointestinal tract. $^{112}$ As stress can induce mast cell degranulation and activation of the central, autonomic, and enteric nervous systems, we postulated that stress might account for some of the pathophysiology observed in certain recurring gut disorders. Therefore, we studied the effects of stress on intestinal physiology.

Male WKY rats were submitted to: restraint stress (RS), a physical type stressor, for two hours, or water avoidance (WA), a psychological stressor, for one hour. Control rats remained in their home cages (for RS groups) or were placed in a waterless tank (for WA group). Subsequently, jejunal and colonic segments were excised, stripped of external muscle, and mounted in Ussing chambers. Short circuit current (Isc), an indicator of ion secretion, conductance (G), an indicator of ionic permeability, and transepithelial fluxes to the small molecule mannitol and to the macromolecule HRP, were measured at 30 minute intervals for two hours. 
In the jejunum, RS, applied either at room temperature or at $8^{\circ} \mathrm{C}$, significantly increased baseline chloride secretion, as indicated by the lack of response in tissues bathed with chloride free buffer compared with controls. ${ }^{13}$ Epithelial permeability to ions and small and large macromolecules was also enhanced in both models of stress, as indicated by the increases in baseline conductance and transepithelial flux of mannitol and HRP..$^{13}$ Similar responses were observed in the colon where both RS and WA significantly increased Isc, conductance, and HRP flux. ${ }^{15}$

Jejunum and colon from stressed animals contained more numerous and dense HRP filled endosomes in all areas of the enterocyte than control tissues. In addition, paracellular passage of HRP was observed only in tissues from stressed animals.

The next step was to identify the mediator(s) of the intestinal responses to stress. Corticotrophin releasing hormone $(\mathrm{CRH})$ is a key hormone that mediates many of the endocrine and behavioural changes induced by stress. We hypothesised that CRH might produce stresslike intestinal epithelial abnormalities. Thus we compared the effects of stress and peripheral $\mathrm{CRH}$ on colonic epithelial function.

CRH $(10 \mu \mathrm{g}$ intraperitoneally) induced significant increases in colonic baseline Isc and $\mathrm{G}$ compared with controls that were similar to those induced by stress. ${ }^{15}$ Both stress and CRH induced a 2-3-fold increase in HRP fluxes compared with their controls. Pretreatment with the $\mathrm{CRH}$ antagonist, a-helical $\mathrm{CRH}_{9-41}(50$ $\mu \mathrm{g}$ intraperitoneally), inhibited the stress and CRH stimulated increases in baseline Isc, G, and HRP flux. This study indicated that peripheral administration of $\mathrm{CRH}$ induced stress-like abnormalities in rat colonic epithelial function that were mediated via activation of specific receptors.

To determine the mechanisms involved in $\mathrm{CRH}$ induced mucosal pathophysiology, different groups of rats were treated with the nicotinic blocker hexamethonium $(15 \mathrm{mg} / \mathrm{kg})$, the muscarinic blocker atropine sulphate (1 $\mathrm{mg} / \mathrm{kg}$ ), the adrenergic blocker bretylium tosylate $(15.8 \mathrm{mg} / \mathrm{kg})$, the steroid synthesis inhibitor aminoglutethimide $(300 \mathrm{mg} / \mathrm{kg})$, or the mast cell stabiliser doxantrazole $(20 \mathrm{mg} / \mathrm{kg})$ prior to administration of $\mathrm{CRH}(10 \mu \mathrm{g})$ or its vehicle. Hexamethonium or atropine abolished the $\mathrm{CRH}$ stimulated increase in baseline Isc whereas bretylium showed no effect. ${ }^{16}$ The increase in colonic $\mathrm{G}$ induced by $\mathrm{CRH}$ was inhibited by hexamethonium or bretylium but was not altered by atropine. Finally, the increase in HRP flux induced by $\mathrm{CRH}$ was significantly reduced by hexamethonium, atropine, or bretylium, all to a similar extent. No changes in epithelial parameters were observed when each drug was administered in control rats.
Treatment with doxantrazole reduced the CRH stimulated increases in baseline Isc and G by $74 \%$ and $73 \%$, respectively. Doxantrazole also reduced HRP flux by $96 \%$ while aminoglutethimide showed no effect on any of these parameters. ${ }^{16}$ Neither doxantrazole nor aminoglutethimide changed Isc, G, or HRP when administered to control rats. This study indicated that CRH induced changes in colonic function involved complex neuroimmune interactions as nicotinic, muscarinic, and adrenergic receptors and mast cells participated in the regulation of these changes.

In conclusion, findings from these series of studies provide strong evidence for the role of stress as a modulator of intestinal and colonic physiology and establish some of the mechanisms involved in these responses. Modulation of stress responses in the gastrointestinal tract involving CRH may be relevant to the management of certain intestinal and colonic disorders.

1 Madara JL, Trier JS. The functional morphology of the mucosa of the small intestine. In: Johnson LR, ed. Physiology of the gastrointestinal tract, 3rd edn. New York: Raven Press, 1994:1577-622.

2 Santos J, Perdue MH. Immunological regulation of intestinal epithelial transport. Digestion 1998;59:404-8.

3 Crowe SE, Sestini P, Perdue MH. Allergic reactions of rat jejunal mucosa. Ion transport responses to luminal challenge and inflammatory mediators. Gastroenterology 1990;99:74-82

4 Perdue MH, Masson S, Wershil BK, et al. Role of mast cells in ion transport abnormalities associated with intestinal anaphylaxis. Correction of diminished secretory response in genetically mast cell deficient W/Wv mice by bone marrow transplantation. 7 Clin Invest 1991;87:687-93.

5 Baird AW, Cuthbert AW, McVinish U. Type 1 hypersensitivity reactions in reconstructed tissues using syngeneic cell types. Br F Pharmacol 1987;91:857-69.

6 Berin MC, Kiliaan AJ, Yang P-C, et al. The influence of mast cells on pathways of transepithelial transport in rat intestine. F Immunol 1998;161:2561-6.

7 Stead RH, Tomioka M, Quinonez G, et al. Intestinal mucosal mast cells in normal and nematode-infected rat intestines are in intimate contact with peptidergic nerves. Proc Natl Acad Sci USA 1987;84:2975-9.

8 MacQueen G, Marshall J, Perdue MH, et al. Pavlovian conditioning of rat mucosal mast cells to secrete rat mast cell protesase II. Science 1989;243:83-5.

9 Crowe SE, Perdue MH. Gastrointestinal food hypersensitivity: basic mechanisms of pathophysiology. Gastroenterology 1992;103:1075-95.

10 Mendeloff Al, Monk M, Siegel CI, et al. Illness experience and life stresses in patients with irritable colon and ulcerative colitis. An epidemiological study of ulcerative colitis and regional enteritis in Baltimore, 1960-1964. N Engl F Med 1970;282:14-17.

11 Barclay GR, Turnberg LA. Effect of psychological stress on salt and water transport in the human jejunum. Gastroenterology 1987;93:91-7.

12 Santos J, Saperas B, Nogueiras C, et al. Release of mast cell mediators into the jejunum by cold pain stress. Gastroenterology 1998;114:640-8.

13 Saunders PR, Kosecka U, McKay DM, et al. Acute stressors stimulate ion secretion and increase epithelial permeability in rat intestine. Am f Physiol 1994;267:G794-9.

14 Kiliaan AJ, Saunders PR, Bijslma PB, et al. Stress stimulates transepithelial macromolecular uptake in rat jejunum. $A m$ f Physiol 1998;275:G1037-44.

15 Santos J, Saunders PR, Yates D, et al. Peripheral corticotropin-releasing hormone increases colonic permeability and ionic secretion through nicotinic pathways and mast cells. Gastroenterology 1998;114:G1684.

16 Santos J, Saunders PR, Yates D, et al. Brain-gut interactions regulate colonic epithelial physiology. Digestion 1988; 59(suppl 3):658. 